\title{
REVIEW ARTICLE PIM kinase inhibition: co-targeted therapeutic approaches in prostate cancer
}

\author{
Sabina Luszczak ${ }^{1}$, Christopher Kumar ${ }^{1}$, Vignesh Krishna Sathyadevan ${ }^{1}$, Benjamin S. Simpson (D) , Kathy A. Gately ${ }^{2}$,
} Hayley C. Whitaker ${ }^{1}$ and Susan Heavey (iD ${ }^{1}$

PIM kinases have been shown to play a role in prostate cancer development and progression, as well as in some of the hallmarks of cancer, especially proliferation and apoptosis. Their upregulation in prostate cancer has been correlated with decreased patient overall survival and therapy resistance. Initial efforts to inhibit PIM with monotherapies have been hampered by compensatory upregulation of other pathways and drug toxicity, and as such, it has been suggested that co-targeting PIM with other treatment approaches may permit lower doses and be a more viable option in the clinic. Here, we present the rationale and basis for cotargeting PIM with inhibitors of PI3K/mTOR/AKT, JAK/STAT, MYC, stemness, and RNA Polymerase I transcription, along with other therapies, including androgen deprivation, radiotherapy, chemotherapy, and immunotherapy. Such combined approaches could potentially be used as neoadjuvant therapies, limiting the development of resistance to treatments or sensitizing cells to other therapeutics. To determine which drugs should be combined with PIM inhibitors for each patient, it will be key to develop companion diagnostics that predict response to each co-targeted option, hopefully providing a personalized medicine pathway for subsets of prostate cancer patients in the future.

Signal Transduction and Targeted Therapy (2020)5:7

; https://doi.org/10.1038/s41392-020-0109-y

\section{INTRODUCTION}

Prostate cancer (PCa) is one of the most common cancers among men, with 1.1 million cases per year worldwide. ${ }^{1}$ As current treatments, ${ }^{2,3}$ including surgery, radiotherapy, chemotherapy, and hormone therapy, result in severe side effects, ${ }^{4}$ the development of new targeted therapies with lower toxicity could significantly improve patient quality of life and potentially extend life. Moreover, neoadjuvant therapeutics effective at reducing tumor volume could potentially allow better preservation of erectile function and urinary continence in radical prostatectomy patients. $^{2,5}$

Tumorigenesis in PCa is often dependent on aberrations in one of the key signal transduction pathways, several of which interact with the PIM family. ${ }^{6}$ The PIM family (proviral integration site for Moloney murine leukemia virus) consists of three serine/threonine kinases, which are known to be overexpressed in PCa, as well as breast cancer and hematological malignancies, and are often correlated with decreased overall survival (OS), resistance to therapy and cancer cell proliferation. ${ }^{7}$ PIM proteins have been implicated in driving cell growth and survival, proliferation, and avoidance of apoptosis ${ }^{7}$ by interacting with other tumorigenic pathways, such as the PI3K (phosphoinositide 3-kinase)/mTOR (mammalian target of rapamycin)/AKT (protein kinase B) pathway, ${ }^{8}$ as well as by influencing oncogenes and tumor suppressor genes. $^{8}$ Moreover, PIM upregulation can cause resistance to conventional chemotherapy, ${ }^{9}$ radiotherapy, ${ }^{10}$ PI3K inhibitors ${ }^{11}$, and other therapeutics. ${ }^{12}$ The activity of PIM is primarily regulated at the transcriptional and protein stabilization level and is mainly influenced by the JAK/STAT (Janus kinase/signal transducer and activator of transcription) pathway, NF-KB (nuclear factor kappa-B) ${ }^{8}$ and HSP90 (heat shock protein 90). ${ }^{13,14}$

This large repertoire of PIM signaling interactions and its implication in resistance to other treatment modalities provide a rationale for co-targeting PIM with other therapies in order to increase its efficacy.

\section{ROLE OF PIM IN PROSTATE CANCER}

The PIM family is composed of three highly conserved serine/ threonine kinases-PIM1, PIM2, and PIM3. PIM1 has been identified to have two isoforms ( 33 and $44 \mathrm{kDa}$ ), PIM2 has three isoforms (34, 37 and $40 \mathrm{kDa}$ ), and PIM3 has one isoform. ${ }^{8}$ Xie et al. suggested that functional differences existed between the long and short isoforms of PIM1, as the $44 \mathrm{kDa}$ isoform (PIM1L) is mainly present on the plasma membrane. and the $33 \mathrm{kDa}$ isoform (PIM1S) is mostly in the nucleus. PIM1L interacts with the SH3 (SRC homology 3) domain of the Etk tyrosine kinase, which has been shown to be one of the sources of resistance to chemotherapeutic drugs in PCa cell models. ${ }^{15}$

The oncogenic potential of the PIM family is perhaps best characterized within PCa, where extensive work has been carried out. Data are available that suggest a role for PIM1 in particular, with higher expression of PIM1 or PIM3 noted in PCa versus matched benign tissues in multiple cohorts. ${ }^{16-20}$ This increase in expression of PIM has prompted numerous studies investigating the role of the whole PIM family in the development and progression of $\mathrm{PCa}{ }^{21}$ Interestingly, the impact of PIM on patient prognosis is disputed, as some reports suggest that low PIM1

\footnotetext{
${ }^{1}$ Molecular Diagnostics and Therapeutics Group, University College London, London, UK and ${ }^{2}$ Trinity Translational Medicine Institute, St. James's Hospital Dublin, Dublin 8, Dublin, Ireland

Correspondence: Susan Heavey (s.heavey@ucl.ac.uk)
}

Received: 24 September 2019 Revised: 5 December 2019 Accepted: 13 December 2019

Published online: 31 January 2020 
expression in prostate cancer can be linked to poor patient outcomes. $^{22}$

PIM1 and PIM2 have been shown to play a role in PCa tumorigenesis, with PIM1 overexpression increasing the tumorigenicity of two PCa cell lines, LNCaP and DU145, both in vitro and in vivo, ${ }^{23}$ while PIM2 has been suggested to play a role in prostate tumorigenesis via phosphorylation of elF4B (eukaryotic translation initiation factor $4 \mathrm{E}){ }^{24}$ It has been noted that this PIM-mediated initiation of prostate tumors is weak and that the kinase family may play a bigger role in the progression of the disease. ${ }^{25} \mathrm{PIM} 1$, in particular, has been implicated in that process, notably contributing to invasion, migration and metastasis in vivo. ${ }^{26,27}$ In transgenic mouse models, PIM1 and PIM2 have also been shown to be overexpressed in prostate tumors that possess inflammatory features and markers of stemness, underpinning their relevance to aggressive, drug-resistant, advanced disease. ${ }^{17}$ PIM2 overexpression is perhaps more clinically important in various hematological cancers, but it has also been shown to be linked to increased proliferation and reduced apoptosis in prostate cancer. ${ }^{28}$ Other hallmarks of cancer that are to some extent influenced by PIM expression include angiogenesis, ${ }^{29}$ migration, and invasion, ${ }^{30-32}$ evasion of the immune response ${ }^{33}$ and modulation of energy metabolism. ${ }^{34,35}$ Unfortunately, most data regarding PIM expression and its clinical impact have been obtained from mouse models and refer mainly to mRNA expression, which may not be an accurate representation of translated kinase levels. This issue could be rectified by employing carefully vetted antibodies to be used in immunohistochemistry analysis of human prostate cancer samples, which would provide researchers with a more accurate representation of human malignancy. ${ }^{36}$

Perhaps PIM's most significant role is in cooperating with other oncogenes, leading to pronounced aggressive phenotypes. One key oncogene that is regulated by PIM is c-MYC (avian myelocytomatosis viral oncogene homolog). Cells expressing both c-MYC and PIM showed increased tumor progression, leading to an advanced cancer phenotype, and silencing of PIM1 resulted in c-MYC-related tumor inactivation, suggesting that PIM1 may be required to maintain C-MYC-driven aggressive $\mathrm{PCa}{ }^{37,38}$ Other downstream PIM targets include p27 (cyclin-dependent kinase inhibitor $1 \mathrm{~B})^{39,40}$ and $\mathrm{BAD}$ (Bcl2-associated death promoter). ${ }^{8,41}$

PIM kinases have also been shown to contribute to the stabilization of the tumor suppressor NKX3.1 by protecting it from proteasome-mediated degradation. Expression of NKX3.1 has been suggested to limit the tumorigenesis of PCa cells and suppress tumor growth. Paradoxically, PIM kinases, despite functioning mainly as oncogenes, protect this prostate-specific tumor suppressor and its anti-tumor activity. Moreover, PIM inhibition reduced NKX3.1 levels. ${ }^{42}$ This relationship emphasizes the complexity of signaling pathways in tumors and suggests that PIM therapy may have a detrimental effect on some tumor suppressors.

\section{PIM INHIBITION AS A MONOTHERAPY}

To date, most efforts to inhibit PIM in cancer treatment have focused on a monotherapeutic approach, generally using ATP (adenosine triphosphate)-competitive drugs that target the kinase action of the protein, preventing it from phosphorylating its downstream effectors, either through quinones or other classes of small molecule inhibitors ${ }^{43-45}$ (Table 1).

AZD1208 is one of the most developed small molecule PIM inhibitors. It is a selective ATP-competitive pan-PIM kinase inhibitor that controls the expression and phosphorylation of downstream PIM effectors such as STAT3 or mTOR. ${ }^{46}$ In PCa, AZD1208 inhibits cell growth, induces apoptosis, inhibits motility and alters cellular morphology, as well as acting as a radiation sensitizer. ${ }^{47}$ A phase I dose-escalation study carried out in 35 solid tumor patients showed that AZD1208 elicited no functional 
response, even though the PIM kinase was successfully inhibited, and it was suggested that the clinical efficacy of the drug might increase in combination with other targeting agents. ${ }^{48}$ Overall, 66 out of 67 patients reported adverse effects posttreatment, including gastrointestinal disorders (nausea and diarrhea) and fatigue. Seventy-five percent of acute myeloid leukemia (AML) patients and $45.7 \%$ of solid cancer patients experienced grade $\geq 3$ adverse effects according to the CTCAE (Common Terminology Criteria for Adverse Effects), including febrile pneumonia, neutropenia, hypotension, and maculopapular rash. Because of the high toxicity, 4 solid tumor patients discontinued their treatment. ${ }^{48}$

TP-3654 (SGI-9481) is a second-generation small molecule panPIM inhibitor with improved potency and decreased cardiotoxicity compared to previous PIM inhibitors. Notably, this drug selectively targets PIM1, with a Ki (inhibition constant) of $5 \mathrm{~nm}$ for PIM1, $239 \mathrm{nM}$ for PIM2 and $42 \mathrm{nM}$ for PIM3. Moreover, it reduced phospho-BAD expression, exhibited no treatment toxicity and reduced tumor volume. ${ }^{49,50}$ This pattern of selectivity is often replicated by other PIM inhibitors, as many of them inhibit PIM2 much less efficiently than other PIM kinases. ${ }^{51}$

A series of quinone analogs have been shown to offer selective inhibition of PIM, leading to attenuation of growth in the PCa cell line DU145. ${ }^{43}$ The PIM selective inhibitor DHPCC-9 impairs the antiapoptotic effects of PIM1 and inhibits the intracellular phosphorylation of PIM substrates, including BAD, leading to reduced invasion and migration in PCa models. ${ }^{30}$ Another small molecule PIM inhibitor, SMI-4a, has been well studied in hematological malignancies, where it has been shown to induce apoptosis and has been recommended for study in PCa models. ${ }^{52}$ CX-6258 is an oxindole-based pan-PIM inhibitor that also shows some effect against FLT-3 (Fms-like tyrosine kinase 3$)^{53}$ and is under preclinical testing, with some evidence to suggest a role in $\mathrm{PCa}$ treatment, especially in combination with other therapeutics such as CX-6461 (refs. ${ }^{32,54}$ ). In AML models, it has been shown to be greatly selective, be well tolerated by mouse carriers, have an anti-proliferative effect, block phosphorylation of BAD and 4E-BP1 (eukaryotic translation initiation factor $4 \mathrm{E}$ binding protein 1), and potentiate the antiproliferative effect of chemotherapeutic agents, such as doxorubicin and paclitaxel. ${ }^{55}$ PIM447 is a selective small molecule pan-PIM inhibitor that has not yet been investigated in $\mathrm{PCa}$; however, the results in large B-cell lymphoma suggest that PIM inhibition by this drug leads to inactivation of the mTORC1 signaling complex, followed by the inactivation of capdependent protein translation, thereby leading to apoptosis and cell death. ${ }^{56}$ This suggests that the known interactions between PIM and PI3K signaling could offer broad signaling effects in response to PIM inhibition as a monotherapy.

Currently, one of the challenges of PIM-targeted therapy is the toxicity of some inhibitors. A clinical trial investigating the efficacy of SGI-1776, a pan-PIM and FLT-3 inhibitor, in prostate cancer and non-Hodgkin lymphoma was terminated early due to its cardiotoxicity. It has been suggested that prolongation of the cardiac QT has been a result of inhibition of the cardiac potassium channel human ether-à-go-go-related gene (hERG). ${ }^{50}$ More novel inhibitors, such as TP-3654 (SGI-9481), have been demonstrated to be better tolerated; however, their effect on hERG and FLT-3 should be evaluated prior to clinical trials to address the issue of potential treatment toxicity.

\section{PIM INHIBITION WITHIN THE PROSTATE CANCER CLINICAL PATHWAY}

Currently, the PCa clinical pathway includes, depending on the risk stratification, active surveillance, surgical prostatectomy, radiotherapy (external beam intensity-modulated and brachytherapy), ${ }^{2}$ hormone therapy, ${ }^{57}$ chemotherapy and radium-223 (ref. ${ }^{3}$ ) (Fig. 1). However, most of these therapies can result in severe side effects and decrease patient quality of life. ${ }^{2,58}$ Successful PIM-targeted therapy could be used as a neoadjuvant drug prior to surgery to shrink the tumor and enable more patients to undergo nervesparing radical prostatectomy and therefore improve functional outcomes. $^{5}$ Moreover, it has been demonstrated that PIM inhibitors can enhance the efficacy of existing PCa treatments, which will be discussed further, including radiotherapy, ${ }^{10}$ chemotherapy, ${ }^{9}$ and androgen deprivation. ${ }^{59}$ PIM co-targeting could also reduce patient mortality by potentiating the antimetastatic effects of other treatments, including strategies targeting the PI3K pathway ${ }^{60,61}$ or JAK/STAT pathway ${ }^{62,63}$ or anti-androgen therapy. ${ }^{64}$

\section{PIM AND THE PI3K PATHWAY}

The PI3K/AKT/mTOR pathway has been shown to contribute to the development of all hallmarks of cancer and is frequently disrupted in cancer. ${ }^{65}$ Three generations of drugs targeting this pathway have been under widespread clinical development for multiple cancers. ${ }^{66}$ However, many have exhibited issues with toxicity or resistance due to compensatory mechanisms and feedback loops with closely related signaling pathways, and co-targeted inhibition approaches have been gaining popularity in preclinical studies. ${ }^{67-70}$ Thus, PIM and PI3K co-targeting may be a viable approach owing to the overlap between those pathways, the proven PIMinduced resistance to PI3K inhibition ${ }^{35}$ and the reported successful synergism that results when both are used. ${ }^{71}$ Synergy between inhibitors could allow the use of lower therapeutic doses to achieve the same clinical effect, thus potentially reducing treatment toxicity and improving patient quality of life.

The PIM and PI3K/AKT/mTOR pathways overlap and influence each other in multiple ways (Fig. 2). PIM mimics the cellular functions of AKT, leading to similar effects on molecules influencing the cell cycle, survival, apoptosis and growth. ${ }^{8}$ This includes the phosphorylation of cell cycle and proliferation mediators, such as p21wafl (cyclin-dependent kinase inhibitor 1A), p27kip (cyclin-dependent kinase inhibitor 1B), and Mdm2 (mouse double minute 2), as well as pro-apoptotic members of the $\mathrm{BCl}-2$ family (B-cell lymphoma gene), such as BAD. Moreover, both PIM and AKT block the caspase-induced apoptotic processes by disrupting caspase 3 and caspase 9 (ref. ${ }^{8}$ ). Another point of convergence of the PIM and PI3K pathways is mTOR, a molecule downstream of PI3K that is responsible for cellular processes, including cell survival and protein synthesis, and influences the pathogenesis of disorders such as cancer and type 2 diabetes. ${ }^{72,73}$ Both PIM and AKT negatively regulate mTOR by phosphorylation of PRAS40 (proline-rich Akt substrate of $40 \mathrm{kDa})^{74}$ and TSC2 (tuberous sclerosis complex 2), ${ }^{75}$ the latter of which occurs by both directly and indirectly decreasing AMPK ( $5^{\prime}$ adenosine monophosphate-activated protein kinase) activation. ${ }^{8}$ Moreover, mTOR and PIM can phosphorylate 4E-BP1 to allow for protein translation and MYC signaling. ${ }^{76}$ Another link between the pathways is the phosphorylation of c-MYC, which prevents apoptosis and allows the oncogene to drive tumorigenesis. ${ }^{7,8,77,78}$

A number of clinical trials have been conducted to investigate the effects of PI3K inhibition in solid tumors, including PCa. The drugs under review in phase I or II trials include BKM120 (NCT01385293, NCT01695473, and NCT02035124), AZD-8186 (NCT01884285 and NCT03218826), GSK2636771 (NCT02215096), PX-866 (NCT01331083) and BEZ-235 (NCT01717898 and NCT01634061). However, many PI3K pathway inhibitors failed to show substantial clinical results as monotherapies, partially owing to the development of toxicity, including autoimmune toxicities, hypertension, neuropsychiatric effects, diarrhea, respiratory complications and susceptibility to infection, and treatment resistance. ${ }^{79,80}$

Resistance mechanisms have been linked to PI3K's ability to downregulate negative feedback loops once inhibited, as well as 


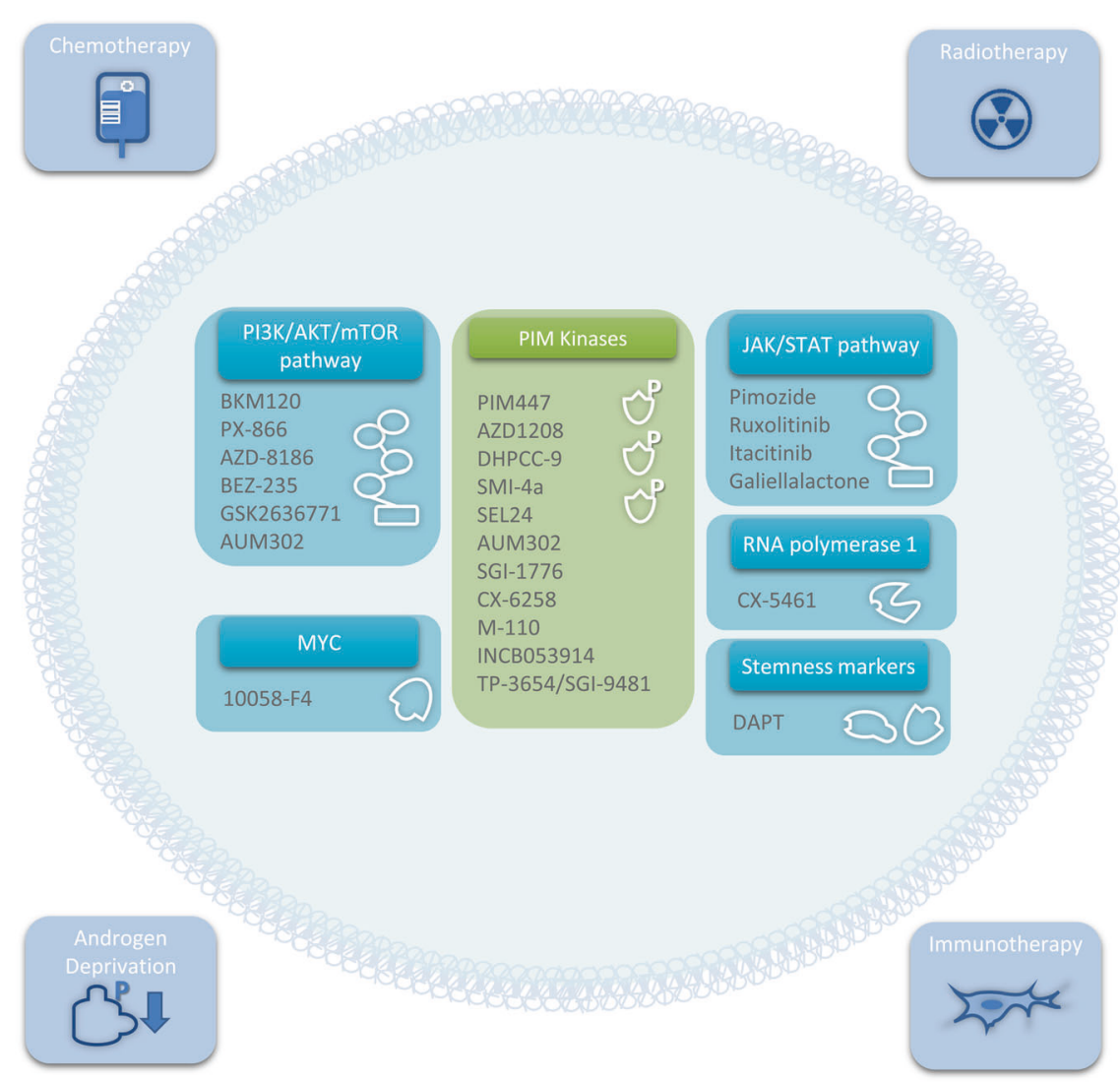

Fig. 1 Categories of therapeutics that could be used in combination with PIM inhibition in prostate cancer. Outer boxes: therapies that are well developed for prostate cancer but that could benefit from the PIM co-targeted inhibition approach. Inner boxes: related targets and specific drugs that are currently under development. Abbreviations: AKT, protein kinase B; EGFR, epidermal growth factor receptor; HER2, human epidermal growth factor receptor 2; mTOR, mammalian target of rapamycin; PI3K, phosphoinositide 3-kinase.

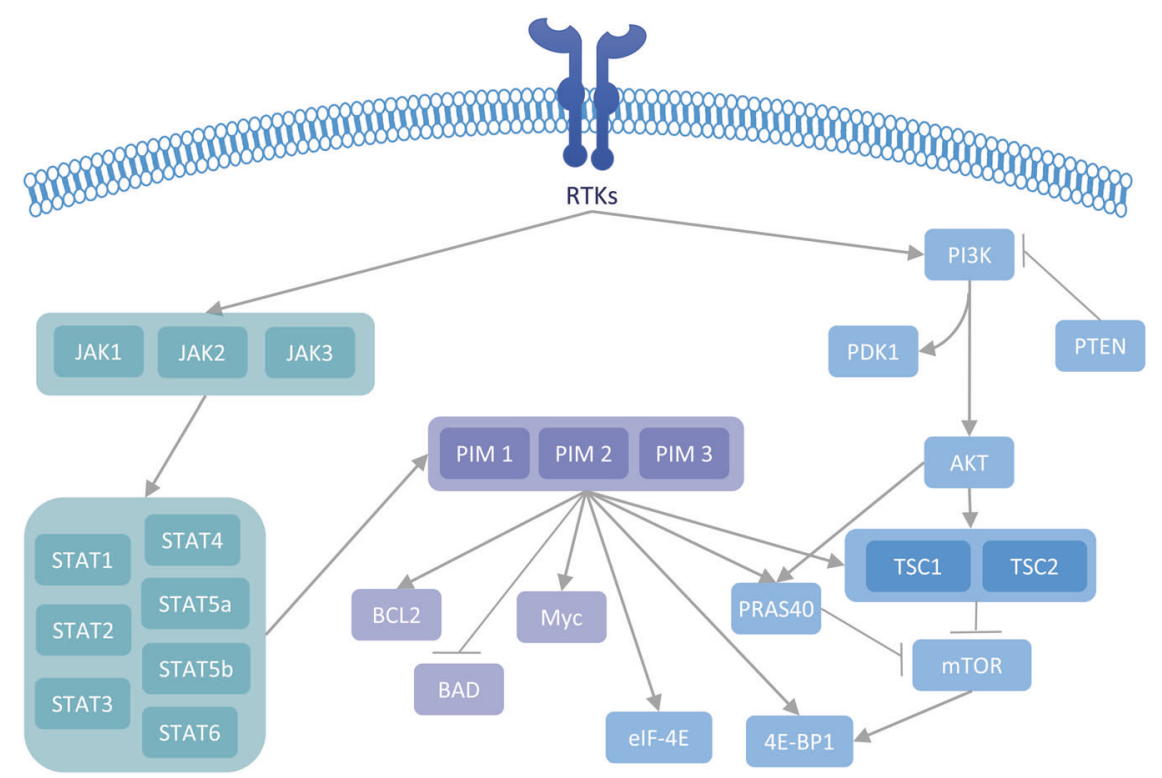

Fig. 2 Key interactions between PIM kinases and targetable signaling pathways. In prostate cancer, the key targetable pathways that interact closely with PIM signaling are the JAK/STAT and PI3K pathways. Abbreviations: 4E-BP1, eukaryotic translation initiation factor 4E binding protein 1; AKT, protein kinase $B$; $B A D, B C l 2$-associated death promoter; $B C L 2$, B-cell CLL/lymphoma 2; elF-4E, eukaryotic translation initiation factor 4E; JAK, Janus kinase; mTOR, mammalian target of rapamycin; MYC, avian myelocytomatosis viral oncogene homolog; PDK1, pyruvate dehydrogenase kinase 1; PI3K, phosphoinositide 3-kinase; PIM, proviral integration site for Moloney murine leukemia virus; PRAS40, proline-rich Akt substrate, $40 \mathrm{KDa}$; PTEN, phosphatase and tensin homolog; RTK, receptor tyrosine kinase; STAT, signal transducer and activator of transcription; TSC, tuberous sclerosis complex. 
to its crosstalk with other signaling pathways including the MAPK (mitogen-activated protein kinase) pathway and the androgen receptor (AR) pathway. ${ }^{79}$ The PIM family has also been implicated in PI3K pathway inhibitor failure, which mainly stems from common substrates affected by both pathways. A suggested mechanism for resistance involves PIM regulation of mTORC1 (ref. ${ }^{35}$ ) and protection of its function despite upstream PI3K inhibition. ${ }^{11}$ FLT3-ITD (internal tandem duplication) has been shown to upregulate STAT5 and subsequently cause PIM overexpression, as well as activate both the PI3K/Akt/mTOR and MAPK pathways, which can all stimulate mTOR signaling. ${ }^{11}$

Efforts have begun to co-target PIM and the PI3K pathway, which has exhibited promising synergistic effects, with the combined therapy suppressing cell growth ${ }^{35,81}$ and viability and increasing apoptosis ${ }^{11}$ in comparison with monotherapies in $\mathrm{PCa}{ }^{71}$ as well as in hematological malignancies. ${ }^{11,82}$

Another study on human PCa cells ${ }^{35}$ supported these findings, showing that combined therapy resulted in lower cell growth and survival than the monotherapies in vitro, as well as higher tumor shrinkage and reduced proliferation in mouse models. The study also presented evidence in favor of the relationship between PIM, mTORC1, NRF2 (nuclear factor erythroid 2-related factor, a molecule involved in response to oxidant stress), ${ }^{83}$ and the resultant mechanisms of $\mathrm{PI} 3 \mathrm{~K}$ inhibitor resistance to apoptosis. Cotargeting PIM and PI3K was more effective than monotherapy in decreasing cell proliferation via accumulation of reactive oxygen species (ROS), a consequence of NRF2 inhibition. As PIM1 increases the synthesis of glutathione, the suppression of both glutathione and PI3K caused a significant increase in ROS accumulation, leading to lower tumor volume; this relationship between PIM1 and glutathione expression could further explain the complex role the PIM family plays in PI3K resistance. ${ }^{83}$

A novel drug targeting PIM/PI3K/mTOR, AUM302 (IBL-302), has recently been reported in neuroblastoma. ${ }^{84}$ It has been shown to be very specific, more bioavailable than earlier drugs of the same pipeline and effective in decreasing levels of downstream targets of PIM/PI3K/mTOR, including phosphorylated AKT and N-Myc. Moreover, AUM302 increased the differentiation of patient-derived xenografts and cancer cell culture models, reduced cell viability and tumor growth, and potentiated the effect of doxorubicin, etoposide, and cisplatin, with the possibility of reducing the dosage of cisplatin by $50 \%$ while retaining its therapeutic effect. In comparison to dactolisib (BEZ-235), a PI3K/mTOR inhibitor, AUM302 was superior in its ability to decrease cell viability. ${ }^{84}$

These initial studies suggest that PIM/PI3K co-targeting has potential clinical benefit, although further in vitro and in vivo experiments should be carried out using different inhibitors prior to PIM-PI3K co-targeted clinical trials.

\section{PIM AND THE JAK/STAT PATHWAY}

PIM is primarily activated by the JAK/STAT signaling pathway, which results in phosphorylation of its downstream targets ${ }^{17}$ (Fig. 2). In response to stimuli such as interleukins, downstream STAT dimers translocate to the nucleus, ${ }^{8}$ where STAT3 and STAT5 have been shown to bind to PIM1 promoters, in particular, promoting aggressive, invasive $\mathrm{PCa}{ }^{85,86}$ It has been suggested that STAT3 does this by aiding myeloid-derived suppressor cells (MDSCs) in their effort to suppress antitumor activity. ${ }^{87}$ STAT3 has been shown to be upregulated in as many as $82 \%$ of PCa tumors compared to surrounding benign tissue. ${ }^{88}$ Moreover, PIM1 upregulation by JAK/STAT activates a negative feedback loop, in which it activates suppressors of cytokine signaling (SOCSs), which prevent JAK/STAT signaling transduction.,89

Given the success of vertical co-targeting approaches in the PI3K pathway, where PI3K and mTOR are frequently co-targeted using dual inhibition strategies, ${ }^{90}$ we should consider the option of vertically co-targeting the PIM pathway with upstream proteins such as STAT3 and STAT5, as opposed to purely horizontal cotargeting approaches with other signaling pathways. Targeting STAT3/STAT5 in PCa has previously been identified as a promising avenue for investigation, with the psychotropic inhibitor pimozide indirectly inhibiting STAT5 and leading to an apoptotic response ${ }^{91}$ and STAT3 inhibition offering promise in PCa treatment. ${ }^{92,93}$ The STAT3-targeted inhibitor galiellalactone has been shown to be successful in preventing the generation of MDSCs and thus suppressing the immune response against PCa cells. ${ }^{87}$ Inhibition of PIM by M-110 and SGI-1776 can indirectly decrease levels of phosphorylated STAT3, but not STAT5, in cell models, which contributed to downregulation of PIM3 (ref. ${ }^{94}$ ). Early work suggests efficacy of the co-targeting strategy in myelofibrosis, leading to a clinical trial combining ruxolitinib, a JAK1/JAK2 inhibitor, with PIM447, a pan-PIM inhibitor ${ }^{95}$ (NCT02370706), and another trial combining JAK1 inhibition (itacitinib) with a preclinical PIM inhibitor (INCB053914) in hematological malignancies. ${ }^{82}$ Contrary to these results, another study found that inactivation of STAT3 or IL-6 (interleukin-6) could lead to quicker PCa progression, as it would prevent STAT3 stimulation of p14 (ARF) and its other tumor suppressor targets. ${ }^{96}$ This evidence would argue that targeting STAT would not be beneficial for the patients, and high levels of STAT3 should be treated as a biomarker and not a therapeutic target. ${ }^{96}$ We propose that a cotargeted approach of inhibiting PIM along with STAT3/5 could potentially offer improved anticancer effects, particularly in the setting of advanced PCa; however, such a strategy should be approached with caution and researched in depth to verify whether it could be a viable treatment option.

\section{PIM AND MYC}

PIM1 and MYC are often coexpressed in PCa and can both influence its development and progress. It has been suggested that PIM itself is only weakly tumorigenic but that it has the ability to potentiate the effect of other oncogenes such as $M^{3} C^{37}$ by increasing its transcriptional activity or stability. ${ }^{38}$ Interestingly, it promotes the oncogenic properties of MYC signaling while suppressing its ability to promote apoptosis. ${ }^{97}$ PIM acts on a large proportion of MYC target genes (41\%), including connective tissue growth factor (CTGF) and CD24 (ref. ${ }^{23}$ ). Coexpression of PIM and MYC has been shown to result in increased proliferation and apoptosis, the latter possibly due to increased cell turnover. ${ }^{37}$ Moreover, PIM and MYC coexpression can be correlated with a higher Gleason grade of $\mathrm{PCa}{ }^{37} \mathrm{MYC}$ promotes prostate tumorigenesis by affecting ribosomal DNA and RNA, activation of protumorigenic genes such as EZH2 (enhancer of zeste homolog 2) and downregulation of tumor suppressor genes such as NKX3.1 (homeobox protein $\mathrm{Nkx}-3.1$ ). ${ }^{97}$

It has been demonstrated that inhibition of c-MYC using 10058F4 suppresses the colony formation of PIM1-expressing PCa cell models (LNCaP and DU145 cells), as well as the expression of PIM1 itself at the protein level. ${ }^{23}$ Interestingly, Wang et al suggested that the effects of PIM1 knockdown on MYC expression varied between different cell lines (DU145 and MPT). ${ }^{38}$ Moreover, PIM inhibitors such as AZD1208 were effective at downregulating MYC activity, inhibiting the growth of MYC-driven PCa, increasing apoptosis and reducing proliferation (BrdU index). ${ }^{47}$ Because of the synergistic effect of PIM and MYC, a co-targeting approach could enhance the effect of the treatment, or alternatively, PIM inhibition could be used to indirectly target the expression of cMyc. $^{23}$ The relationship between PIM and MYC is one of the examples of synthetic lethality and how it can be used to maximize the efficacy of existing drugs via inhibition of proteins related to the effector of interest. ${ }^{98}$ Other targets that exhibit synthetic lethality with PIM could be identified by genetic screening, which would uncover the epigenetic changes driving tumorigenesis in prostate cancer. 


\section{PIM AND STEMNESS}

PIM1 has been shown to play a role in the development of stemness phenotypes. In mouse embryonic and rat and ovine mesenchymal stem cells, PIM1 is upregulated, which may lead to self-renewal, increased proliferation and survival. ${ }^{99-101}$ Human cardiac stem/progenitor cells with transduced PIM1 exhibited lower senescence, longer telomeres, and higher telomerase activity, proliferation and cell survival than control cells. ${ }^{102,103}$ Moreover, PIM1 has been found to act on putative stem cell markers, including breast cancer resistance protein (BCRP)/ adenosine triphosphate-binding cassette subfamily $\mathrm{G}$ member 2 (ABCG2), leading to its membrane translocation and development of resistance to chemotherapeutics. ${ }^{59,104}$ BCRP/ABCG2 has also been shown to guard prostate stem-like cells from the negative effects of androgen deprivation, hypoxia, and chemotherapeutic drugs, which encourages the development of PCa. ${ }^{104}$ As PIM1 may stimulate a stem-like phenotype within prostate cancer, targeting it could be effective in preventing progression in the early stages of the disease.

PIM kinases have been implicated in the Notch (neurogenic locus Notch homolog protein) embryonic pathway, which plays a role in the development of various cancers, including prostate and hematological malignancies, ${ }^{105}$ by inducing epithelial-tomesenchymal transition and promoting resistance to chemotherapy and survival. ${ }^{106,107}$ PIM kinases have been shown to phosphorylate and stimulate the activity of Notch1, and activated Notch1 can upregulate the expression of PIM. ${ }^{107}$ Moreover, PIM kinases and Notch share some downstream targets, including MYC, p21, and NF-KB. ${ }^{108}$ It has been shown that inhibition of PIM (via the drug DHPCC-9) or Notch (via the drug DAPT) alone led to a reduction of tumor volume in PCa cell models; however, a co-targeting treatment approach proved more effective than any of the monotherapies and could be of interest for patients with aberrations in the PIM or Notch signaling pathways. ${ }^{107}$

\section{PIM AND RNA POLYMERASE I TRANSCRIPTION}

MYC has been shown to drive cell growth by upregulating ribosomal RNA synthesis. As mentioned previously, PIM is often coexpressed with MYC and can stimulate its transcriptional activity and stability, as well as act on other downstream targets, such as $4 \mathrm{E}-\mathrm{BP} 1$, to contribute to PCa tumorigenesis and growth. Inhibition of RNA polymerase I, crucial for ribosome biogenesis, together with PIM kinases could synergistically block MYC signaling and thus produce better functional effects than targeting either alone. A study on PCa cell models has shown their sensitivity to treatment with inhibitors of RNA polymerase I and PIM (CX-5461 and CX-6258, respectively), with both drugs suppressing colony formation and causing cell cycle arrest. ${ }^{32}$ The effects of the treatments were potentiated with the combined therapies compared to those seen with either agent alone. Moreover, cotreatment with CX-5461 and CX-6258 in vivo reduced the tumor growth and proliferation and increased the apoptosis of patientderived xenografts from castration-resistant PCa patients, which suggests that this combined approach could also be effective in patients with advanced diseases resistant to most current therapies. $^{32}$ Similarly, the efficacy of CX-5461 together with CX6258 in prostate ex vivo patient-derived xenografts has been demonstrated by another study, as the drugs, alone or in combination, were effective in all four types of diverse tumors; these results contrast with those achieved with other therapeutics, including the PARP (poly(ADP-ribose) polymerase) inhibitor talazoparib and the CDK4-6 (cyclin-dependent kinase) inhibitors ribociclib or cisplatin, which were effective against one or none of the samples. ${ }^{54}$ Moreover, the co-targeting approach upregulated the DNA damage response and downregulated downstream signaling from PIM and mTORC1 (ref. ${ }^{54}$ ).

\section{PIM AND ANDROGEN DEPRIVATION THERAPY (ADT)}

PIM1 is known to phosphorylate AR, which plays a key role in $\mathrm{PCa}$ progression. $^{21,109}$ Both PIM1S and PIM1L interact with AR, and differential phosphorylation of $A R$ at multiple sites leads to modulation of stability and transcriptional activity. ${ }^{110}$ PIMmediated phosphorylation of the receptor was observed in both the presence and absence of the hormone; this process was decreased upon treatment with PIM inhibitors. ${ }^{109}$ PIM is linked to castration-resistant PCa (CRPC) through pathways associated with cMET (tyrosine-protein kinase Met), MYC and Oct4 (octamer-binding transcription factor 4), pathways believed to act as compensatory mechanisms in androgen deprivation therapy (ADT). ${ }^{59}$

PIM1 expression is maintained during androgen deprivation therapy in mice, implying that it may be important for maintenance of proliferation in this setting. ${ }^{111}$ AR levels do not appear to affect PIM1, which would suggest a promising approach for co-targeting, as the two are independently expressed. Investigations combining PIM inhibition and ADT are needed to assess whether the inhibition of PIM could overcome resistance to ADT.

\section{PIM AND EGFR}

EGFR (epidermal growth factor receptor) overexpression is commonly correlated with the development of many cancers, including prostate cancer. ${ }^{112}$ PIM1 expression is correlated with EGFR expression in head and neck cancer, in which anti-EGFR treatment (cetuximab and gefitinib) prevented the EGFRstimulated translocation of PIM1 (ref. ${ }^{113}$ ). In breast cancer, PIM1 has been implicated in driving resistance to HER2 (human epidermal growth factor receptor 2) via inactivation of $B A D$, thereby allowing cells to escape the effect of the drugs. ${ }^{114}$

In prostate cancer cell models, the PIM inhibitors M-110 and SGI-1776 successfully upregulated the expression of MIG6 (mitogen-inducible gene 6 protein), which prevents EGFR signaling. M-110 has also been shown to prevent EGF-stimulated activation and reduce the expression of EGFR, leading to lower ERK (extracellular signal-regulated kinase) pathway activity. Moreover, co-targeting PIM and EGFR using M-110 or SGI-1776 with gefitinib showed a synergistic effect on cell proliferation. ${ }^{112}$ This combination approach has also been shown in non-small-cell lung cancer (NSCLC), where AZD-1208 and osimertinib (an EGFR inhibitor) elicited synergistic effects with respect to cell viability and phosphorylation of STAT3 (ref. ${ }^{115}$ ), which, if replicated in PCa, could prevent STAT3-driven promotion of aggressive prostate cancer features. $^{86}$

\section{PIM AND RADIOTHERAPY}

PCa radiotherapy resistance is often linked to and may be attributed to aberrations in the expression of various signaling pathways and genes involved in cell growth and cell death, including downregulation of DOC2/DAB2 (double C2 domain; DAB adaptor protein 2) and P53 (tumor protein P53) and upregulation of MDM2 (ref. ${ }^{116}$ ), epidermal growth factor (EGF), BCL-2 (ref. ${ }^{117}$ ), and the $\mathrm{PISK} / \mathrm{Akt} / \mathrm{mTOR}$ pathway. ${ }^{118-120}$ Other factors contributing to radioresistance may include cancer stem cells ${ }^{121,122}$ and the tumor microenvironment. ${ }^{120}$ Recently, PIM has also been implicated in contributing to tumor radioresistance. ${ }^{10,123-125}$

Upregulation of PIM has been noted following exposure to radiation, which leads to PIM translocation into the nucleus, phosphorylation of PRAS40 and formation of a complex of phospho-PRAS40, 14-3-3 protein and phospho-FOXO3a (forkhead box O3), which accumulates in the cytoplasm. This accumulation could lead to resistance to therapy and low levels of proapoptotic genes. ${ }^{125}$ PIM inhibition disallows phosphorylation of PRAS40 and results in increased nuclear FOXO3a, which increases the level of pro-apoptotic proteins (e.g., Fas and Fas ligand) and reduces 
radioresistance. $^{10}$ Increased expression of PIM1 correlates with reduced efficacy of radiotherapy and increased expression of EGFR, which has been shown to act on PIM1 and stimulate its nuclear translocation. ${ }^{113}$

A study by Kirschner et al. investigated the relationship between radiation and PIM expression as well as the effect of a PIM inhibitor (AZD1208) on PCa radioresistance. ${ }^{47}$ They showed that radiation and hypoxia increased the expression of PIM1, which in turn led to increased efficacy of the drug. This approach was also effective at reducing tumor recurrence. Similar results, showing an encouraging synergistic effect of combining PIM inhibition and radiotherapy, have been reported for pancreatic cancer. $^{126}$

\section{PIM AND CHEMOTHERAPY}

PCa studies have suggested a potential role for PIM1 in the development of acquired resistance to chemotherapeutic agents, ${ }^{9,15}$ with further data supporting this role in other cancers such as hematopoietic malignancies ${ }^{127}$ and NSCLC. ${ }^{128}$ This overexpression is likely contributing to increased survival signaling during treatment. ${ }^{9}$ Cytotoxic drugs such as docetaxel induce significant PIM overexpression in RWPE-2 benign prostate epithelial cells at the protein (as much as 6.25 -fold) and mRNA (2-4-fold) levels. ${ }^{9}$ This may be caused by a simultaneous increase in phospho-STAT3, which upregulates PIM.,129

Knock-out studies in PCa cell models (PCA3 cell line) suggested that the absence of PIM increases apoptotic cell death, which becomes more prominent after exposure to chemotherapeutic anti-tubulin agents such as paclitaxel, vincristine, evodiamine or colchicine. ${ }^{129}$ However, this effect was not dependent on tubulin polymerization but rather on blocking DNA repair mechanisms via inhibition of DNA kinases such as ATM (ataxia-telangiectasia mutated kinase) or DNA-PK (DNA-dependent protein kinase) and upregulating $\mathrm{H} 2 \mathrm{~A} . \mathrm{X}$ ( $\mathrm{H} 2 \mathrm{~A}$ histone family member $\mathrm{X}$ ) phosphorylation, which is involved in DNA fragmentation as a part of the apoptotic mechanism. Moreover, similar results regarding an increase in H2A.X phosphorylation and PARP-1 cleavage as well as reduced ATM and RPA32 (replication protein A $32 \mathrm{kDa}$ subunit) phosphorylation were demonstrated when a PIM inhibitor, quercetin, was used together with paclitaxel in PIM-expressing cells. ${ }^{129}$

Further evidence that PIM1 overexpression protects tumor cells from chemotherapy-induced apoptosis is that its downregulation leads to increased sensitivity to treatment. ${ }^{9}$ This effect may be mediated by NF-KB signaling, particularly by the RELA (nuclear factor NF-kappa-B P65 subunit) component of the complex. ${ }^{9}$ Upregulation of NF-KB itself has been shown to weaken the proapoptotic effects of chemotherapeutic agents such as paclitaxel, likely by affecting MDR1 (multidrug resistance protein 1) and other anti-apoptotic genes. ${ }^{130,131}$ Moreover, as mentioned above, PIM1$\mathrm{L}$ and its target BCRP are both overexpressed in mitoxantroneand docetaxel-resistant PCa, and PIM knockdown has been shown to restore sensitivity to chemotherapy in affected cells. ${ }^{104}$

Another study on PIM inhibitors and chemotherapeutic agents in PCa suggests that a combined therapy involving paclitaxel and PIM inhibition has a synergistic effect on reducing cell viability, affecting cell cycle distribution and reducing apoptosis. ${ }^{132}$

\section{PIM AND IMMUNOTHERAPY}

Upstream molecules modifying the expression and function of PIM include interleukins such as IL-2 (interleukin-2), IL-3 (interleukin-3) and IL-6. As such, it has been suggested that PIM could be targeted indirectly using immunotherapeutics. In PCa cell models, an IL-6 antibody has been shown to decrease PIM1 expression. ${ }^{15}$ Moreover, PIM1-specific antibodies decreased prostate tumor and leukemia cell growth, increased apoptosis and affected levels of PIM1, HSP90, AKT and caspase pathway proteins. $^{133}$ PIM kinases have been shown to phosphorylate glycogen synthase kinase $3 \beta$ (GSK3B) and the tumor suppressor FOXP3 and block their influence on prostate cancer cells, leading to increased cell migration and adhesion. ${ }^{134}$ Moreover, FOXP3 has been shown to be involved in the development of $T$ regulatory cells (T-regs), ${ }^{135}$ which have been implicated in driving tumor resistance to immunotherapeutics. ${ }^{136,137}$ Targeting T-regs with a PIM1 inhibitor helped to improve their suppressive activity. ${ }^{135,136}$ Kaempferol, a plant flavonoid with PIM1-inhibitory properties, has been shown to exhibit protective functions against high-dose IL-2induced toxicity due to its positive effect on T-regs. Treatment with kaempferol led to lower body weight loss and higher survival than the control treatment. ${ }^{138}$ In Hodgkin lymphoma, PIM kinases have been shown to contribute to the immunosuppressive environment through modulation of PD-L1/2 (programmed death-ligand) activity, and although the PIMs are not key drivers of this phenotype, there is some rationale to support investigating the co-targeting of the PIM family (via the pan-PIM inhibitor SEL24) with PDL-1 (139). A recent study on adoptive T-cell therapy (ACT) has suggested that PIM inhibition could be used alongside anti-PD1 (programmed death receptor 1) therapy. ${ }^{139}$ The cells obtained from PIM knock-out mice had lower glycolytic activity, S6 phosphorylation, interferon gamma secretion, and ROS levels than those from wild-type mice, which translates to decreased T-cell death, an increased memory phenotype, and superior tumor control and mouse survival. A similar outcome was demonstrated following treatment with AZD1208. These effects were potentiated by the use of an anti-PD1 antibody (triple combination therapy), prolonging mouse survival, decreasing tumor growth and improving T-cell migration. ${ }^{140}$ Multiple studies have suggested the use of immunotherapeutics, perhaps alongside PIM inhibitors, to overcome the mechanisms of resistance to treatment and improve the functional outcomes. ${ }^{27,133}$

\section{CONCLUDING REMARKS}

Inhibition of PIM in PCa has attracted much attention as a potential monotherapeutic over the last decade, with growing in vitro, in vivo and early clinical trial data supporting this approach to varying degrees. However, despite extensive investigation, no PIM inhibitors have yet been approved for patient treatment in PCa. Targeting PIM alone has been suggested to be ineffective because of the existence of signaling bypass tracks that could lead to acquired resistance and because inhibitor toxicity limits the safe and tolerable dose. Utilizing a combined therapeutic approach, with both PIM inhibitors and other signal transduction inhibitors or other standard PCa treatments, could overcome these issues, reducing toxicity and improving patient quality of life and potentially survival. These benefits have already been demonstrated for some combinations in vitro and in vivo, and clinical trials are warranted to determine potential patient benefits. Following a demonstration of the efficacy of such strategies, it will be crucial to identify predictive companion biomarkers that can determine which patient groups are likely to benefit from each combination therapy and thus adopt a more personalized approach to treatment, matching the appropriate combination with the appropriate patient. This could be achieved by using proteomics ${ }^{141}$ or RNA sequencing ${ }^{142}$ in patients treated with the specific therapies. In summary, we believe that cotargeting PIM with rational treatment combinations is a promising concept for improved treatment of PCa.

\section{ACKNOWLEDGEMENTS}

The authors would like to thank Prostate Cancer UK for funding SH under a Travelling Prize Fellowship (TLD-PF16-004) and acknowledge the support of the Prostate Cancer UK Centre of Excellence. 


\section{ADDITIONAL INFORMATION}

Competing interests: The authors declare no competing interests.

\section{REFERENCES}

1. Stewart, B. W. \& Wild, C. P. World Cancer Report 2014 (International Agency for Research on Cancer 2014).

2. van Stam, M. A. et al. Patient-reported Outcomes Following Treatment of Localised Prostate Cancer and Their Association with Regret About Treatment Choices. Eur. Urol. Oncol. https://doi.org/10.1016/j.euo.2018.12.004 (2018).

3. Tucci, M. et al. Therapeutic options for first-line metastatic castration-resistant prostate cancer: Suggestions for clinical practise in the CHAARTED and LATITUDE era. Cancer Treat. Rev. 74, 35-42 (2019).

4. James, N. D. et al. Addition of docetaxel, zoledronic acid, or both to first-line long-term hormone therapy in prostate cancer (STAMPEDE): survival results from an adaptive, multiarm, multistage, platform randomised controlled trial. Lancet 387, 1163-1177 (2016).

5. Imyanitov, E. N. \& Yanus, G. A. Neoadjuvant therapy: theoretical, biological and medical consideration. Chin. Clin. Oncol. 7, 55 (2018).

6. Brault, L. et al. PIM serine/threonine kinases in the pathogenesis and therapy of hematologic malignancies and solid cancers. Haematologica 95, 1004-1015 (2010).

7. Rebello, R. J., Huglo, A. V. \& Furic, L. PIM activity in tumours: A key node of therapy resistance. Adv. Biol. Regul. 67, 163-169 (2018).

8. Warfel, N. A. \& Kraft, A. S. PIM kinase (and Akt) biology and signaling in tumors. Pharm. Ther. 151, 41-49 (2015).

9. Zemskova, M., Sahakian, E., Bashkirova, S. \& Lilly, M. The PIM1 kinase is a critical component of a survival pathway activated by docetaxel and promotes survival of docetaxel-treated prostate cancer cells. J. Biol. Chem. 283, 20635-20644 (2008).

10. Kim, W. et al. PIM1 kinase inhibitors induce radiosensitization in non-small cell lung cancer cells. Pharm. Res. 70, 90-101 (2013).

11. Okada, K. et al. FLT3-ITD induces expression of Pim kinases through STAT5 to confer resistance to the PI3K/Akt pathway inhibitors on leukemic cells by enhancing the mTORC1/Mcl-1 pathway. Oncotarget 9, 8870-8886 (2018).

12. Green, A. S. et al. Pim kinases modulate resistance to FLT3 tyrosine kinase inhibitors in FLT3-ITD acute myeloid leukemia. Sci. Adv. 1, e1500221 (2015).

13. Shay, K. P., Wang, Z., Xing, P. X., McKenzie, I. F. \& Magnuson, N. S. Pim-1 kinase stability is regulated by heat shock proteins and the ubiquitin-proteasome pathway. Mol. Cancer Res. 3, 170-181 (2005).

14. Toren, P. \& Zoubeidi, A. Rational cotargeting of Pim-1 and Akt in prostate cancer. Expert Rev. Anticancer Ther. 13, 937-939 (2013).

15. Xie, Y. et al. The 44 kDa Pim-1 kinase directly interacts with tyrosine kinase Etk/ BMX and protects human prostate cancer cells from apoptosis induced by chemotherapeutic drugs. Oncogene 25, 70-78 (2006).

16. He, H. C. et al. Real-time quantitative RT-PCR assessment of PIM-1 and hK2 mRNA expression in benign prostate hyperplasia and prostate cancer. Med Oncol. 26, 303-308 (2009).

17. Jimenez-Garcia, M. P. et al. The role of PIM1/PIM2 kinases in tumors of the male reproductive system. Sci. Rep. 6, 38079 (2016)

18. Tomlins, S. A. et al. Integrative molecular concept modeling of prostate cancer progression. Nat. Genet 39, 41-51 (2007).

19. Qu, Y. et al. Pim-3 is a critical risk factor in development and prognosis of prostate cancer. Med Sci. Monit. 22, 4254-4260 (2016).

20. Taylor, B. S. et al. Integrative genomic profiling of human prostate cancer. Cancer Cell 18, 11-22 (2010).

21. Holder, S. L. \& Abdulkadir, S. A. PIM1 kinase as a target in prostate cancer: roles in tumorigenesis, castration resistance, and docetaxel resistance. Curr. Cancer Drug Targets 14, 105-114 (2014).

22. Dhanasekaran, S. M. et al. Delineation of prognostic biomarkers in prostate cancer. Nature 412, 822-826 (2001).

23. Kim, J., Roh, M. \& Abdulkadir, S. A. Pim1 promotes human prostate cancer cell tumorigenicity and c-MYC transcriptional activity. BMC Cancer 10, 248 (2010).

24. Ren, K. et al. The over-expression of Pim-2 promote the tumorigenesis of prostatic carcinoma through phosphorylating elF4B. Prostate 73, 1462-1469 (2013).

25. Narlik-Grassow, M. et al. Conditional transgenic expression of PIM1 kinase in prostate induces inflammation-dependent neoplasia. PLOS ONE 8, e60277 (2013).

26. Santio, N. M. et al. Pim kinases promote migration and metastatic growth of prostate cancer xenografts. PLOS ONE 10, e0130340 (2015).

27. Tursynbay, Y. et al. Pim-1 kinase as cancer drug target: an update. Biomed. Rep. 4, 140-146 (2016).

28. Dai, H. et al. Pim-2 upregulation: biological implications associated with disease progression and perinueral invasion in prostate cancer. Prostate 65, 276-286 (2005).
29. Casillas, A. L. et al. Hypoxia-inducible PIM kinase expression promotes resistance to antiangiogenic agents. Clin. Cancer Res. 24, 169-180 (2018).

30. Santio, N. M. et al. Pim-selective inhibitor DHPCC-9 reveals Pim kinases as potent stimulators of cancer cell migration and invasion. Mol. Cancer 9, 279 (2010).

31. Liu, J. et al. Pim-3 enhances melanoma cell migration and invasion by promoting STAT3 phosphorylation. Cancer Biol. Ther. 19, 160-168 (2018).

32. Rebello, R. J. et al. The dual inhibition of RNA Pol I transcription and PIM kinase as a new therapeutic approach to treat advanced prostate cancer. Clin. Cancer Res. 22, 5539-5552 (2016).

33. Jinesh, G. G., Mokkapati, S., Zhu, K. \& Morales, E. E. Pim kinase isoforms: devils defending cancer cells from therapeutic and immune attacks. Apoptosis 21, 1203-1213 (2016).

34. Zhang, X. H., Yu, H. L., Wang, F. J., Han, Y. L. \& Yang, W. L. Pim-2 modulates aerobic glycolysis and energy production during the development of colorectal tumors. Int J. Med. Sci. 12, 487-493 (2015).

35. Song, J. H. et al. Mechanisms behind resistance to $\mathrm{PI} 3 \mathrm{~K}$ inhibitor treatment induced by the PIM kinase. Mol. Cancer Ther. 17, 2710-2721 (2018).

36. Sfanos, K. S. et al. If this is true, what does it imply? How end-user antibody validation facilitates insights into biology and disease. Asian J. Urol. 6, 10-25 (2019).

37. Wang, J. et al. Pim1 kinase synergizes with c-MYC to induce advanced prostate carcinoma. Oncogene 29, 2477-2487 (2010).

38. Wang, J. et al. Pim1 kinase is required to maintain tumorigenicity in MYCexpressing prostate cancer cells. Oncogene 31, 1794-1803 (2012).

39. Morishita, D., Katayama, R., Sekimizu, K., Tsuruo, T. \& Fujita, N. Pim kinases promote cell cycle progression by phosphorylating and down-regulating p27Kip1 at the transcriptional and posttranscriptional levels. Cancer Res. 68, 5076-5085 (2008).

40. Santio, N. M. \& Koskinen, P. J. PIM kinases: From survival factors to regulators of cell motility. Int. J. Biochem. Cell. Biol. 93, 74-85 (2017).

41. Aho, T. L. et al. Pim-1 kinase promotes inactivation of the pro-apoptotic Bad protein by phosphorylating it on the Ser112 gatekeeper site. FEBS Lett. 571, 43-49 (2004).

42. Padmanabhan, A., Gosc, E. B. \& Bieberich, C. J. Stabilization of the prostatespecific tumor suppressor NKX3.1 by the oncogenic protein kinase Pim-1 in prostate cancer cells. J. Cell Biochem. 114, 1050-1057 (2013).

43. Schroeder, R. L. et al. Identification of quinones as novel PIM1 kinase inhibitors. Bioorg. Med. Chem. Lett. 26, 3187-3191 (2016).

44. Mohare, R. M., Abbas, N. S. \& Wardakhan, W. W. Synthesis of tetrahydrobenzo[b] thiophene-3-carbohydrazide derivatives as potential anti-cancer agents and Pim-1 kinase Inhibitors. Anticancer Agents Med. Chem. https://doi.org/10.2174/ 1871520619666190402153429 (2019).

45. Wang, X. \& Sun, Z. Understanding PIM-1 kinase inhibitor interactions with free energy simulation. Phys. Chem. Chem. Phys. 21, 7544-7558 (2019).

46. Yadav, A. K., Kumar, V., Bailey, D. B. \& Jang, B. C. AZD1208, a Pan-Pim kinase inhibitor, has anti-growth effect on 93T449 human liposarcoma cells via control of the expression and phosphorylation of Pim-3, mTOR, 4EBP-1, S6, STAT-3 and AMPK. Int. J. Mol. Sci. 20, https://doi.org/10.3390/ijms20020363 (2019).

47. Kirschner, A. N. et al. PIM kinase inhibitor AZD1208 for treatment of MYC-driven prostate cancer. J. Natl Cancer Inst. 107, https://doi.org/10.1093/jnci/dju407 (2015).

48. Cortes, J. et al. Phase I studies of AZD1208, a proviral integration Moloney virus kinase inhibitor in solid and haematological cancers. Br. J. Cancer 118, 1425-1433 (2018).

49. Le, B. T. et al. Targeting Pim kinases for cancer treatment: opportunities and challenges. Future Med. Chem. 7, 35-53 (2015).

50. Foulks, J. M. et al. A small-molecule inhibitor of PIM kinases as a potential treatment for urothelial carcinomas. Neoplasia 16, 403-412 (2014).

51. Cervantes-Gomez, F. et al. PIM kinase inhibitor, AZD1208, inhibits protein translation and induces autophagy in primary chronic lymphocytic leukemia cells. Oncotarget 10, 2793-2809 (2019).

52. Kuang, X. et al. PIM inhibitor SMI-4a induces cell apoptosis in B-cell acute lymphocytic leukemia cells via the HO-1-mediated JAK2/STAT3 pathway. Life Sci. 219, 248-256 (2019).

53. Bogusz, J. et al. Structural analysis of PIM1 kinase complexes with ATPcompetitive inhibitors. Sci. Rep. 7, 13399 (2017).

54. Lawrence, M. G. et al. Patient-derived models of abiraterone- and enzalutamideresistant prostate cancer reveal sensitivity to ribosome-directed therapy. Eur. Urol. 74, 562-572 (2018).

55. Haddach, M. et al. Discovery of CX-6258. A potent, selective, and orally efficacious pan-Pim Kinases Inhibitor. ACS Med Chem. Lett. 3, 135-139 (2012).

56. Peters, T. L., Li, L., Tula-Sanchez, A. A., Pongtornpipat, P. \& Schatz, J. H. Control of translational activation by PIM kinase in activated B-cell diffuse large B-cell lymphoma confers sensitivity to inhibition by PIM447. Oncotarget 7, 63362-63373 (2016) 
57. Crawford, E. D. et al. Androgen-targeted therapy in men with prostate cancer: evolving practice and future considerations. Prostate Cancer Prostatic Dis. 22, 24-38 (2019).

58. Hall, F. et al. Estimating utilities/disutilities for high-risk metastatic hormonesensitive prostate cancer ( $\mathrm{mHSPC}$ ) and treatment-related adverse events. Qual. Life Res. 28, 1191-1199 (2019).

59. Xie, Y. \& Bayakhmetov, S. PIM1 kinase as a promise of targeted therapy in prostate cancer stem cells. Mol. Clin. Oncol. 4, 13-17 (2016).

60. Liu, J. et al. In vitro and in vivo antimetastatic effects of ZSTK474 on prostate cancer DU145 Cells. Curr. Cancer Drug Targets 19, 321-329 (2019).

61. Zhao, W. et al. In vitro antimetastatic effect of phosphatidylinositol 3-kinase inhibitor ZSTK474 on prostate cancer PC3 cells. Int. J. Mol. Sci. 14, 13577-13591 (2013).

62. Kim, B. H. et al. Development of oxadiazole-based ODZ10117 as a smallmolecule inhibitor of STAT3 for targeted cancer therapy. J. Clin. Med. 8, https:// doi.org/10.3390/jcm8111847 (2019).

63. Kim, J. W., Gautam, J., Kim, J. E., Kim, J. A. \& Kang, K. W. Inhibition of tumor growth and angiogenesis of tamoxifen-resistant breast cancer cells by ruxolitinib, a selective JAK2 inhibitor. Oncol. Lett. 17, 3981-3989 (2019).

64. Rashid, M. et al. Efficacy and safety of non-steroidal anti-androgens in patients with metastatic prostate cancer: meta-analysis of randomized controlled trials. Rev. Recent Clin. Trials. https://doi.org/10.2174/1574887114666191105152404 (2019).

65. Janku, F., Yap, T. A. \& Meric-Bernstam, F. Targeting the PI3K pathway in cancer: are we making headway? Nat. Rev. Clin. Oncol. 15, 273-291 (2018).

66. Yang, J. et al. Targeting PI3K in cancer: mechanisms and advances in clinical trials. Mol. Cancer 18, 26 (2019).

67. Heavey, S., O'Byrne, K. J. \& Gately, K. Strategies for co-targeting the PI3K/AKT/ mTOR pathway in NSCLC. Cancer Treat. Rev. 40, 445-456 (2014).

68. Heavey, S. et al. Strategic targeting of the PI3K-NFkappaB axis in cisplatinresistant NSCLC. Cancer Biol. Ther. 15, 1367-1377 (2014).

69. Heavey, S. et al. In pursuit of synergy: an investigation of the PI3K/mTOR/MEK co-targeted inhibition strategy in NSCLC. Oncotarget 7, 79526-79543 (2016).

70. Heavey, S. et al. Development and characterisation of a panel of phosphatidylinositide 3-kinase - mammalian target of rapamycin inhibitor resistant lung cancer cell lines. Sci. Rep. 8, 1652 (2018).

71. Mologni, L., Magistroni, V., Casuscelli, F., Montemartini, M. \& Gambacorti-Passerini, C. The novel PIM1 inhibitor NMS-P645 reverses PIM1-dependent effects on TMPRSS2/ERG positive prostate cancer cells and shows anti-proliferative activity in combination with PI3K inhibition. J. Cancer 8, 140-145 (2017).

72. Laplante, M. \& Sabatini, D. M. mTOR signaling in growth control and disease. Cell 149, 274-293 (2012).

73. Qin, X., Jiang, B. \& Zhang, Y. 4E-BP1, a multifactor regulated multifunctional protein. Cell Cycle 15, 781-786 (2016).

74. Zhang, F. et al. PIM1 protein kinase regulates PRAS40 phosphorylation and mTOR activity in FDCP1 cells. Cancer Biol. Ther. 8, 846-853 (2009).

75. Lu, J. et al. Pim2 is required for maintaining multiple myeloma cell growth through modulating TSC2 phosphorylation. Blood 122, 1610-1620 (2013).

76. Keane, N. A., Reidy, M., Natoni, A., Raab, M. S. \& O'Dwyer, M. Targeting the Pim kinases in multiple myeloma. Blood Cancer J. 5, e325 (2015).

77. Amaravadi, R. \& Thompson, C. B. The survival kinases Akt and Pim as potential pharmacological targets. J. Clin. Invest. 115, 2618-2624 (2005).

78. Zhang, Y., Wang, Z., Li, X. \& Magnuson, N. S. Pim kinase-dependent inhibition of c-Myc degradation. Oncogene 27, 4809-4819 (2008).

79. Park, S., Kim, Y. S., Kim, D. Y., So, I. \& Jeon, J. H. PI3K pathway in prostate cancer: all resistant roads lead to PI3K. Biochim Biophys. Acta Rev. Cancer 1870, 198-206 (2018).

80. Greenwell, I. B., Ip, A. \& Cohen, J. B. PI3K Inhibitors: Understanding toxicity mechanisms and management. Oncol. (Williston Park) 31, 821-828 (2017).

81. Cen, B., Mahajan, S., Wang, W. \& Kraft, A. S. Elevation of receptor tyrosine kinases by small molecule AKT inhibitors in prostate cancer is mediated by Pim-1. Cancer Res. 73, 3402-3411 (2013).

82. Koblish, H. et al. Preclinical characterization of INCB053914, a novel pan-PIM kinase inhibitor, alone and in combination with anticancer agents, in models of hematologic malignancies. PLOS ONE 13, e0199108 (2018).

83. $\mathrm{Ma}, \mathrm{Q}$. Role of nrf2 in oxidative stress and toxicity. Annu Rev. Pharm. Toxicol. 53 401-426 (2013).

84. Mohlin, S. et al. Anti-tumor effects of PIM/PI3K/mTOR triple kinase inhibitor IBL302 in neuroblastoma. EMBO Mol. Med. 11, e10058 (2019).

85. Gu, L. et al. Stat 5 promotes metastatic behavior of human prostate cancer cells in vitro and in vivo. Endocr. Relat. Cancer 17, 481-493 (2010).

86. Horinaga, M. et al. Clinical and pathologic significance of activation of signal transducer and activator of transcription 3 in prostate cancer. Urology $\mathbf{6 6}$ 671-675 (2005).
87. Hellsten, R., Lilljebjorn, L., Johansson, M., Leandersson, K. \& Bjartell, A. The STAT3 inhibitor galiellalactone inhibits the generation of MDSC-like monocytes by prostate cancer cells and decreases immunosuppressive and tumorigenic factors. Prostate, https://doi.org/10.1002/pros.23885 (2019).

88. Mora, L. B. et al. Constitutive activation of Stat3 in human prostate tumors and cell lines: direct inhibition of Stat3 signaling induces apoptosis of prostate cancer cells. Cancer Res. 62, 6659-6666 (2002).

89. Narlik-Grassow, M., Blanco-Aparicio, C. \& Carnero, A. The PIM family of serine/ threonine kinases in cancer. Med. Res. Rev. 34, 136-159 (2014).

90. Roccaro, A. M. et al. Dual targeting of the PI3K/Akt/mTOR pathway as an antitumor strategy in Waldenstrom macroglobulinemia. Blood 115, 559-569 (2010).

91. Mohanty, S. K. et al. STAT3 and STAT5A are potential therapeutic targets in castration-resistant prostate cancer. Oncotarget 8, 85997-86010 (2017).

92. Barton, B. E., Karras, J. G., Murphy, T. F., Barton, A. \& Huang, H. F. Signal transducer and activator of transcription 3 (STAT3) activation in prostate cancer: Direct STAT3 inhibition induces apoptosis in prostate cancer lines. Mol. Cancer Ther. 3, 11-20 (2004).

93. Thaper, D. et al. Galiellalactone inhibits the STAT3/AR signaling axis and suppresses Enzalutamide-resistant prostate cancer. Sci. Rep. 8, 17307 (2018).

94. Chang, M. et al. PIM kinase inhibitors downregulate STAT3(Tyr705) phosphorylation. Mol. Cancer Ther. 9, 2478-2487 (2010).

95. Mazzacurati, L. et al. The PIM inhibitor AZD1208 synergizes with ruxolitinib to induce apoptosis of ruxolitinib sensitive and resistant JAK2-V617F-driven cells and inhibit colony formation of primary MPN cells. Oncotarget 6, 40141-40157 (2015).

96. Pencik, J. et al. STAT3 regulated ARF expression suppresses prostate cancer metastasis. Nat. Commun. 6, 7736 (2015).

97. Rebello, R. J., Pearson, R. B., Hannan, R. D. \& Furic, L. Therapeutic approaches targeting MYC-driven prostate cancer. Genes (Basel) 8, https://doi.org/10.3390/ genes8020071 (2017).

98. Horiuchi, D. et al. PIM1 kinase inhibition as a targeted therapy against triplenegative breast tumors with elevated MYC expression. Nat. Med. 22, 1321-1329 (2016).

99. Aksoy, l. et al. Self-renewal of murine embryonic stem cells is supported by the serine/threonine kinases Pim-1 and Pim-3. Stem Cells 25, 2996-3004 (2007).

100. Tu, M. L. et al. Pim-1 is up-regulated by shear stress and is involved in shear stress-induced proliferation of rat mesenchymal stem cells. Life Sci. 88, 233-238 (2011).

101. Zhao, Y. et al. Pim-1 kinase cooperates with serum signals supporting mesenchymal stem cell propagation. Cells Tissues Organs 199, 140-149 (2014).

102. Mohsin, S. et al. Rejuvenation of human cardiac progenitor cells with Pim-1 kinase. Circ. Res. 113, 1169-1179 (2013).

103. Linn, D. E. et al. A Role for OCT4 in Tumor Initiation of Drug-Resistant Prostate Cancer Cells. Genes Cancer 1, 908-916 (2010).

104. Xie, Y. et al. The 44-kDa Pim-1 kinase phosphorylates BCRP/ABCG2 and thereby promotes its multimerization and drug-resistant activity in human prostate cancer cells. J. Biol. Chem. 283, 3349-3356 (2008).

105. Ahmed, A. A. et al. Targeted Notch1 inhibition with a Notch 1 antibody, OMPA2G1, decreases tumor growth in two murine models of prostate cancer in association with differing patterns of DNA damage response gene expression. $J$. Cell Biochem. https://doi.org/10.1002/jcb.28954 (2019).

106. Amin, A. et al. Evasion of anti-growth signaling: A key step in tumorigenesis and potential target for treatment and prophylaxis by natural compounds. Semin Cancer Biol. 35 Suppl, S55-S77 (2015).

107. Santio, N. M. et al. Phosphorylation of Notch1 by Pim kinases promotes oncogenic signaling in breast and prostate cancer cells. Oncotarget 7, 43220-43238 (2016).

108. Nefedova, Y. \& Gabrilovich, D. Mechanisms and clinical prospects of Notch inhibitors in the therapy of hematological malignancies. Drug Resist Updat 11 210-218 (2008).

109. $\mathrm{Ha}, \mathrm{S}$. et al. Phosphorylation of the androgen receptor by PIM1 in hormone refractory prostate cancer. Oncogene 32, 3992-4000 (2013).

110. Linn, D. E. et al. Differential regulation of androgen receptor by PIM-1 kinases via phosphorylation-dependent recruitment of distinct ubiquitin E3 ligases. J. Biol. Chem. 287, 22959-22968 (2012).

111. Wang, J. et al. Androgen deprivation therapy has no effect on Pim-1 expression in a mouse model of prostate cancer. Oncol. Lett. 13, 4364-4370 (2017).

112. Siu, A., Virtanen, C. \& Jongstra, J. PIM kinase isoform specific regulation of MIG6 expression and EGFR signaling in prostate cancer cells. Oncotarget 2, 1134-1144 (2011).

113. Peltola, K. et al. Pim-1 kinase expression predicts radiation response in squamocellular carcinoma of head and neck and is under the control of epidermal growth factor receptor. Neoplasia 11, 629-636 (2009). 
114. Moody, S. E. et al. PRKACA mediates resistance to HER2-targeted therapy in breast cancer cells and restores anti-apoptotic signaling. Oncogene 34, 2061-2071 (2015).

115. Bracht, J. W. P. et al. PIM-1 inhibition with AZD1208 to prevent osimertinibinduced resistance in EGFR-mutation positive non-small cell lung cancer. J Cancer Metastasis Treat. 5, 22 (2019).

116. Impicciatore, G., Sancilio, S., Miscia, S. \& Di Pietro, R. Nutlins and ionizing radiation in cancer therapy. Curr. Pharm. Des. 16, 1427-1442 (2010).

117. An, J., Chervin, A. S., Nie, A., Ducoff, H. S. \& Huang, Z. Overcoming the radioresistance of prostate cancer cells with a novel Bcl-2 inhibitor. Oncogene 26, 652-661 (2007).

118. Chen, Y. A. et al. Antrocin sensitizes prostate cancer cells to radiotherapy through inhibiting PI3K/AKT and MAPK signaling pathways. Cancers (Basel) 11, https://doi.org/10.3390/cancers11010034 (2018).

119. Chang, L. et al. Acquisition of epithelial-mesenchymal transition and cancer stem cell phenotypes is associated with activation of the PI3K/Akt/mTOR pathway in prostate cancer radioresistance. Cell Death Dis. 4, e875 (2013).

120. Alberti, C. Prostate cancer: radioresistance molecular target-related markers and foreseeable modalities of radiosensitization. Eur. Rev. Med. Pharm. Sci. 18, 2275-2282 (2014).

121. Jiang, W., Peng, J., Zhang, Y., Cho, W. C. \& Jin, K. The implications of cancer stem cells for cancer therapy. Int. J. Mol. Sci. 13, 16636-16657 (2012).

122. Rycaj, K. \& Tang, D. G. Cancer stem cells and radioresistance. Int. J. Radiat. Biol. 90, 615-621 (2014).

123. Chen, X. Y., Wang, Z., Li, B., Zhang, Y. J. \& Li, Y. Y. Pim-3 contributes to radioresistance through regulation of the cell cycle and DNA damage repair in pancreatic cancer cells. Biochem Biophys. Res. Commun. 473, 296-302 (2016).

124. $\mathrm{Gu}, \mathrm{H}$. et al. Hypoxia-responsive miR-124 and miR-144 reduce hypoxia-induced autophagy and enhance radiosensitivity of prostate cancer cells via suppressing PIM1. Cancer Med. 5, 1174-1182 (2016).

125. Kim, W. et al. PIM1-activated PRAS40 regulates radioresistance in non-small cell lung cancer cells through interplay with FOXO3a, 14-3-3 and protein phosphatases. Radiat. Res. 176, 539-552 (2011).

126. $\mathrm{Xu}, \mathrm{D}$. et al. The oncogenic kinase Pim-1 is modulated by K-Ras signaling and mediates transformed growth and radioresistance in human pancreatic ductal adenocarcinoma cells. Carcinogenesis 32, 488-495 (2011).

127. Pircher, T. J., Zhao, S., Geiger, J. N., Joneja, B. \& Wojchowski, D. M. Pim-1 kinase protects hematopoietic FDC cells from genotoxin-induced death. Oncogene 19, 3684-3692 (2000).

128. Henry, R. E. et al. Acquired savolitinib resistance in non-small cell lung cancer arises via multiple mechanisms that converge on MET-independent MTOR and MYC activation. Oncotarget 7, 57651-57670 (2016).

129. Hsu, J. L. et al. Pim-1 knockdown potentiates paclitaxel-induced apoptosis in human hormone-refractory prostate cancers through inhibition of NHEJ DNA repair. Cancer Lett. 319, 214-222 (2012).

130. Flynn, V. Jr. et al. Adenovirus-mediated inhibition of NF-kappaB confers chemosensitization and apoptosis in prostate cancer cells. Int. J. Oncol. 23, 317-323 (2003).
131. Davoudi, Z. et al. Molecular target therapy of AKT and NF-kB signaling pathways and multidrug resistance by specific cell penetrating inhibitor peptides in HL-60 cells. Asian Pac. J. Cancer Prev. 15, 4353-4358 (2014).

132. Mori, M. et al. A combination strategy to inhibit Pim-1: synergism between noncompetitive and ATP-competitive inhibitors. ChemMedChem 8, 484-496 (2013).

133. Hu, X. F. et al. PIM-1-specific mAb suppresses human and mouse tumor growth by decreasing PIM-1 levels, reducing Akt phosphorylation, and activating apoptosis. J. Clin. Invest. 119, 362-375 (2009).

134. Santio, N. M. et al. The PIM1 kinase promotes prostate cancer cell migration and adhesion via multiple signalling pathways. Exp. Cell Res. 342, 113-124 (2016).

135. Deng, G. et al. Pim-2 kinase influences regulatory $T$ cell function and stability by mediating Foxp3 protein N-terminal phosphorylation. J. Biol. Chem. 290, 20211-20220 (2015).

136. Li, Z. et al. PIM1 kinase phosphorylates the human transcription factor FOXP3 at serine 422 to negatively regulate its activity under inflammation. J. Biol. Chem. 289, 26872-26881 (2014).

137. Nie, $\mathrm{H}$. et al. Phosphorylation of FOXP3 controls regulatory T cell function and is inhibited by TNF-alpha in rheumatoid arthritis. Nat. Med. 19, 322-328 (2013).

138. Li, Y. et al. Regulatory $\mathrm{T}$ cells control toxicity in a humanized model of IL-2 therapy. Nat. Commun. 8, 1762 (2017).

139. Szydlowski, M. et al. Expression of PIM kinases in Reed-Sternberg cells fosters immune privilege and tumor cell survival in Hodgkin lymphoma. Blood 130, 1418-1429 (2017).

140. Chatterjee, S. et al. Targeting PIM kinase with PD1 inhibition improves immunotherapeutic antitumor T-cell response. Clin. Cancer Res. 25, 1036-1049 (2019).

141. Sahab, Z. J., Semaan, S. M. \& Sang, Q. X. Methodology and applications of disease biomarker identification in human serum. Biomark. Insights 2, 21-43 (2007).

142. Akond, Z., Alam, M. \& Mollah, M. N. H. Biomarker identification from RNA-Seq data using a Robust statistical approach. Bioinformation 14, 153-163 (2018).

(i) Open Access This article is licensed under a Creative Commons Attribution 4.0 International License, which permits use, sharing, adaptation, distribution and reproduction in any medium or format, as long as you give appropriate credit to the original author(s) and the source, provide a link to the Creative Commons license, and indicate if changes were made. The images or other third party material in this article are included in the article's Creative Commons license, unless indicated otherwise in a credit line to the material. If material is not included in the article's Creative Commons license and your intended use is not permitted by statutory regulation or exceeds the permitted use, you will need to obtain permission directly from the copyright holder. To view a copy of this license, visit http://creativecommons. org/licenses/by/4.0/.

(c) The Author(s) 2020 